\title{
A Decrement Method for Quantifying Nonlinear and Linear Damping in Multidegree of Freedom Systems
}

\author{
Craig Meskell \\ School of Engineering, Trinity College, Dublin 2, Ireland \\ Correspondence should be addressed to Craig Meskell, cmeskell@tcd.ie
}

Received 18 January 2011; Accepted 13 February 2011

Academic Editors: S. Marchesiello and R. Nagaosa

Copyright () 2011 Craig Meskell. This is an open access article distributed under the Creative Commons Attribution License, which permits unrestricted use, distribution, and reproduction in any medium, provided the original work is properly cited.

\begin{abstract}
A method is presented which can estimate the linear and nonlinear damping parameters in a lightly damped multidegree of freedom system which allows the system to be decomposed into a set of single degree of freedom nonlinear systems. Only a single response measurement from a free decay test is required as input ensuring that the magnitude of the damping parameters is not compromised by phase distortion between measurements. The response is band-pass filtered in the time domain, around each of the natural frequencies. While this provides a free response measurement for each mode, it introduces a restriction as the natural frequencies must be distinct and separated. The instantaneous energy of each trace is used to describe the long-term evolution of the mode. This is achieved by using only the peak amplitudes in each period, and so the stiffness and inertial forces are effectively ignored, and only the damping forces are considered. Thus, the method is not unlike the familiar decrement method, which can estimate the viscous damping in linear systems. The method is developed for a weakly nonlinear, lightly damped two-degree-offreedom system, with both linear and Coulomb damping. Simulated response data is used to demonstrate the accuracy of the technique.
\end{abstract}

\section{Introduction}

Weak nonlinear damping can play a crucial role in the longterm behaviour of weakly nonlinear systems. For example, in self-excited systems, such as fluidelastic systems where the structural and fluid mechanics are strongly coupled, the onset of instability (flutter) is governed largely by the total linear damping in the system, but the amplitude of the resulting self-excited limit cycle oscillations is determined by the nonlinear damping in the system. This has been shown experimentally for tube arrays by Meskell and Fitzpatrick [1], where the nonlinear damping has been found to be cubic in nature. In structural systems, the most likely form of nonlinear damping is Coulomb damping which can be difficult to quantify due to uncertainty in the supports. In this case, simply using an effective linearised damping is unlikely to yield accurate predictions of vibration amplitude under different excitation, as the relative effect of the Coulomb damping will drop as response amplitude increases. Therefore, a quantified nonlinear system model would allow improved predictions of vibration response amplitude which is an issue for accurate long-term fatigue estimates. However, for many structural and fluid-coupled systems (e.g., heat exchanger tubes, long pipework), the total structural damping is very low. Identifying the functional form of nonlinear damping and quantifying the relevant coefficient in lightly damped systems, such as the those discussed above, can be problematic because of the relatively small contribution of the terms of interest to the overall force acting on the structure. Sophisticated system identification procedures for nonlinear system do exist. For example, in the frequency domain, nonlinear spectral estimation has been described by Rice and Fitzpatrick [2] and widely applied to various nonlinear systems. Granger [3] developed an estimation technique essentially based on a nonlinear optimisation of a data model composed of damped sinusoidal basis functions (and so the scheme was nonlinear in the identification parameters) and applied the method specifically to fluidelastic systems. In the time domain, the force surface mapping technique [4-7] has been successfully applied to lightly damped fluidelastic systems [8]. A recent survey of identification methods [9] cites mainly systems 
exhibiting relatively strong nonlinearities. However, these approaches, and many not listed, are not robust when the term of interest has a low contribution. Furthermore, if more than one measurement instrument is required (e.g., in a forced response test or in the case of force surface mapping), then the value of damping coefficients obtained is extremely sensitive to small relative phase distortion between the signals [10].

Meskell [11] proposed a new technique applicable to weak nonlinear systems with a single degree of freedom. This method is similar to the decrement method used to determine the logarithmic decrement and requires only a single input signal. As this signal is a deterministic response, ensemble averaging in the time domain can be used to reduce the effect of noise and extraneous excitation. While it is true that a decrement method for systems with viscous and Coulomb damping has been developed by Feeny and Liang [12], this technique is limited to Coulomb damping only. A general identification procedure for $n$th power damping was developed by Mottershead and Stanway [13], but as the stiffness is identified as part of the procedure, weak nonlinear damping terms cannot be reliably obtained. The method proposed by Meskell has the advantage that it can be applied to a system with low damping of any functional form. Furthermore, Eret and Meskell [14] have shown that it can be successfully applied to a fluidelastic system, and the requirement to know the functional form a priori can be resolved by first applying the FREEVIB method of Feldman [15]. However, one of the limitations is that the method is applicable only to a single degree of freedom system. This paper presents an extension of the approach to a system with multiple degrees of freedom. Band-pass filters applied to a single response measurement provides a technique for decomposing a multi-degree-offreedom system with light linear and nonlinear damping into a number of uncoupled single-degree-of-freedom systems with both linear and nonlinear damping.

The enhanced method is demonstrated with a numerical simulation of a discrete two-degree-of-freedom system with Coulomb damping as well as linear damping.

\section{Extension to 2 Degrees of Freedom}

At the outset, it should be emphasized that the type of system of interest is lightly damped and has only weak nonlinearity. As the systems are lightly damped, only the response at the natural frequencies will be significant. Thus, the objective is not to isolate the nonlinearity but rather to account for the effect of the nonlinear damping on the system response at each natural frequency.

In order to investigate the applicability of the nonlinear decrement method to a system with more than one degree of freedom, consider the two-degree-of-freedom system shown in Figure 1. The system is linear except for a weak nonlinear damping directly coupling the two masses. For the purposes of this study, this nonlinearity is a Coulomb damper.

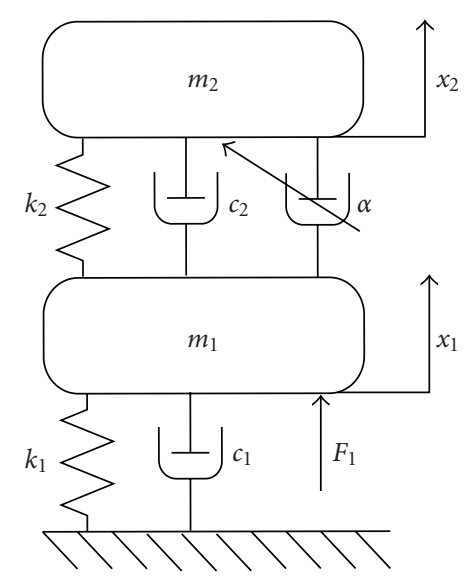

FIGURE 1: Schematic of nonlinear 2-degree-of-freedom system.

As the system is nonlinear, it is convenient to consider the discussion in the context of a specific example. The system parameters used in this study are specified in Tables 1 and 2.

Ignoring the damping (both linear and nonlinear), the natural frequencies of the system (i.e., the eigen frequencies) are $6.9 \mathrm{~Hz}$ and $18.2 \mathrm{~Hz}$; the modal matrix is

$$
v=\left[\begin{array}{cc}
0.74 & -1.20 \\
1.20 & 0.74
\end{array}\right] .
$$

As the linear and nonlinear damping are small compared to the stiffness and inertial forces, the frequencies of vibration will be almost identical to the eigen frequencies.

2.1. Modal Decomposition. For a linear system (i.e., $\alpha=0$ ), if the viscous damping is proportional (i.e., a linear combination of the mass and stiffness matrices), then the system could be decomposed exactly into two uncoupled single-degree-of-freedom systems. However, it can be easily shown that if the damping is not proportional, but the system is lightly damped, then the modal decomposition of the undamped system offers a good estimate of both the natural frequencies and the apparent mode shapes of the damped system. As the total damping is low and the nonlinearities are weak, it is assumed that this engineering approach can be applied to the nonlinear system. In other words, it is assumed that the system shown in Figure 1 can be replaced with two mass-normalized single-degree-offreedom systems, each with a weak nonlinearity as shown in Figure $2(i=1,2)$.

Note that the nonlinear parameter in each mode, $\alpha_{i}$, is also mass normalized, as is the modal excitation force, $f_{i}$. This will necessitate that the modal matrix (rather than just the mode ratios) for the undamped system is known. While this is not a trivial requirement, as it requires some knowledge of the distribution of mass and stiffness in the system, it is not impractical.

While the natural frequencies $\omega_{i}$ can easily be obtained directly from the system response, the other modal parameters are more difficult. 


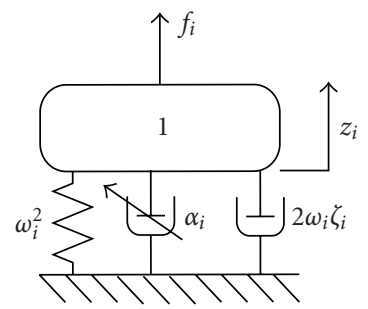

Figure 2: Mass-normalized mode.

TABLe 1: System mass and stiffness values.

\begin{tabular}{lccc}
\hline$m_{1}(\mathrm{~kg})$ & $m_{2}(\mathrm{~kg})$ & $k_{1}(\mathrm{~N} / \mathrm{m})$ & $k_{2}(\mathrm{~N} / \mathrm{m})$ \\
\hline 0.5 & 0.5 & 2500 & 2500 \\
\hline
\end{tabular}

TABLE 2: System damping parameters.

\begin{tabular}{lcc}
\hline$c_{1}(\mathrm{Ns} / \mathrm{m})$ & $c_{2}(\mathrm{Ns} / \mathrm{m})$ & $\alpha(\mathrm{N})$ \\
\hline 1.5 & 2.0 & 0.5 \\
\hline
\end{tabular}

If the system is disturbed from equilibrium, so that the initial displacements are not in the ratio of only one mode, then the free response will exhibit both natural frequencies and hence both modes. Obviously, if both system responses $\left(x_{1}\right.$ and $\left.x_{2}\right)$ are recorded, the modal matrix can be used to construct the normal coordinates and hence achieve the modal decoupling envisaged by Figure 2 . The system parameters could then be obtained using parameter identification techniques developed for SDOF systems. However, it may not be practical to measure all the responses directly if the system has more than two degrees of freedom, and even if it is possible, magnitude and phase distortion in the instruments are likely to lead to substantial errors in estimating the damping parameters. Thus, it is desirable to obtain the damping parameters of each mode of the system from just a single response measurement. Given one measured free response of the system to a disturbance, say $x_{1}$, the problem is now to decompose that response into the uncoupled modal responses.

2.2. Mode Separation. As the system is lightly damped, the system free response in the frequency domain will be confined to narrow bands at the natural frequencies. If the natural frequencies are distinct, the response at each natural frequency, and hence at each mode, can be easily separated using a series of band-pass filters. As the signal is inherently transient and the relatively long-term evolution of the response is of primary concern, this filtering can best be achieved in the time domain.

Figure 3 shows the free response $x_{1}$. It is apparent that the response contains vibration at both natural frequencies.

Two third-order elliptic filters with a pass band ripple of $1 \mathrm{~dB}$ and a stop-band attenuation of $50 \mathrm{~dB}$, with narrow pass bands $4 \mathrm{~Hz}$ wide centered on each natural frequency, are used. A practical issue is that the filter itself will introduce transients, which is caused by the nonzero initial conditions. This can be seen in Figure 4. Obviously, it would be impossible to estimate the damping from such a signal.

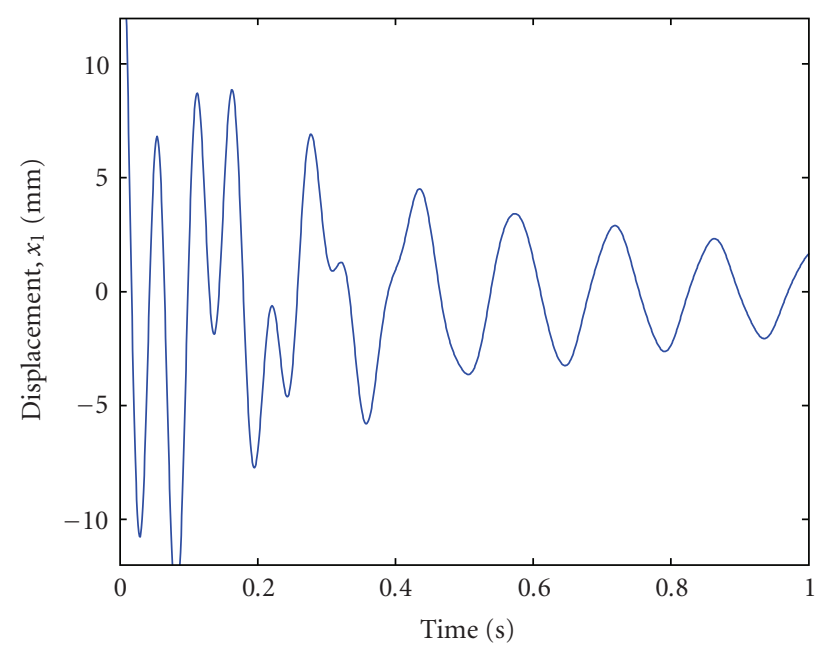

FIgURE 3: Free response of mass 1.

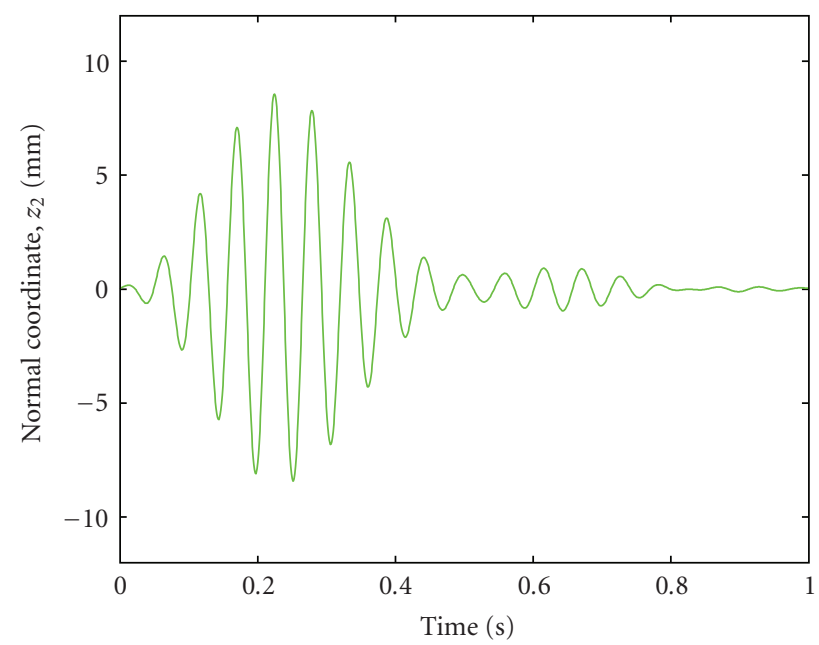

FIgURE 4: Free response corrupted by filter transient.

As the filtering is applied off line, the entire time record is available, and so a simple strategy can be employed to negate this filter transient. As the time record of the free response inherently decays to zero, simply reversing the order of the time record before filtering and reversing again afterwards removes any filter characteristics from the area of interest (i.e., close to $t=0$ ). The separated modes obtained from this processing is shown in Figure 5.

In practice, the acceleration is often the quantity measured, as accelerometers are reliable, robust, and relatively cheap. As the acceleration has been band-passed filtered, it is straightforward to numerically integrate the acceleration trace to obtain the displacement. The filtering process ensures that the low frequency noise often associated with numerical integration does not arise.

\section{Parameter Identification Method}

Once the normal coordinates have been extracted, the system response has been decomposed into that of two singledegree-of-freedom systems. Thus, the method proposed by 


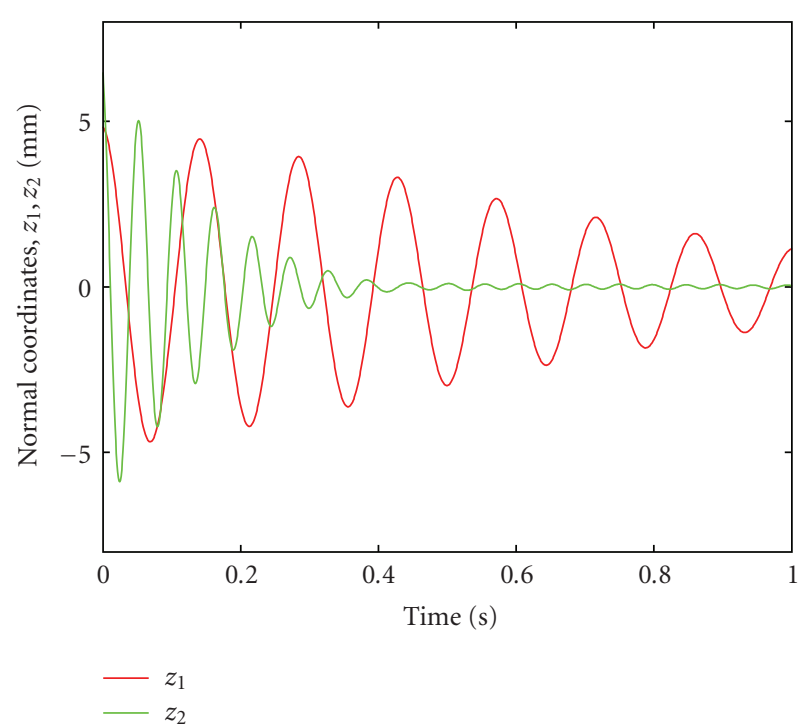

FIGURE 5: Free response of each mode.

Meskell [11] for a single-degree-of-freedom system can be applied directly. For completeness, the technique is briefly presented below and the formulation for a system with Coulomb damping is developed.

3.1. General Formulation. Consider a single-degree-of-freedom system which has a linear spring and a general nonlinear element. The system is in free response, and so no external force is acting on it. The method proposed here makes use of successive peaks in the free response of the system to indicate the rate of energy dissipation and hence the relative strength of the various damping components.

The mass normalized equation of motion is

$$
\ddot{x}+\omega^{2} x+f_{D}(x, \dot{x})=0,
$$

where $\omega=\sqrt{\mathrm{k} / \mathrm{m}}$ and $f_{D}$ is the mass-normalizeddamping force, including both linear and nonlinear damping. Assuming that nonlinear forces are relatively small, the free response can be assumed to be a simple sinusoid with a time-varying amplitude

$$
x(t) \approx A(t) \sin \left(\omega_{d} t+\phi\right)
$$

In other words, the sub- and superharmonics which would be expected from nonlinear components are neglected. Furthermore, if the damping is now assumed to be small in comparison to the stiffness and inertial forces, the rate of energy dissipation must be small, and so the rate of change of the amplitude of vibration is also small (i.e., $\dot{A} \approx 0$ ). In addition, the frequency of vibration $\omega_{d} \approx \omega$. Using these assumptions, the response of the system can now be written as

$$
\begin{gathered}
x=A(t) \sin (\omega t+\phi), \\
\dot{x}=A(t) \omega \cos (\omega t+\phi), \\
\ddot{x}=-A(t) \omega^{2} \sin (\omega t+\phi) .
\end{gathered}
$$

The mass normalized (specific) instantaneous mechanical energy in the system

$$
e(t)=\frac{1}{2} \omega^{2} x^{2}(t)+\frac{1}{2} \dot{x}^{2}(t)
$$

Substituting (4) into this relates the instantaneous specific energy to the instantaneous amplitude of vibration

$$
e(t)=\frac{1}{2} \omega^{2} A^{2}(t)
$$

Alternatively, the specific energy can be obtained by considering the energy dissipated by the nonconservative (i.e., damping) force $f_{d}$. The instantaneous energy is simply

$$
e(t)=e_{0}(t)-w(t)
$$

where $e_{0}$ is the initial energy at $t=0$. The specific work done by the damping force is

$$
w(t)=\int_{0}^{t} f_{D}(\tau) \dot{x}(\tau) d \tau
$$

Combining (6), (7), and (8) yields

$$
A^{2}(t)=A_{0}^{2}-\frac{2}{\omega^{2}} \int_{0}^{t} f_{D}(\tau) \dot{x}(\tau) d \tau,
$$

where $A_{0}$ is the amplitude at $t=0$. Substituting the system velocity (4), changing the variable in the integral to $\theta=$ $\omega \tau+\phi$, and evaluating this equation over one period of vibration yields the basic equation for the decrement method employed

$$
A_{1}^{2}=A_{0}^{2}-\frac{2}{\omega^{3}} \int_{0}^{2 \pi} f_{D}(\theta) \dot{x}(\theta) d \theta .
$$

The values $A_{0}$ and $A_{1}$ are successive peak amplitudes in the response signal for the system undergoing a free decay from an initial perturbation. The damping force $f_{D}(\theta)$ and response $\dot{x}(\theta)$ depends on the instantaneous amplitude $A(\theta)$, but as the system is lightly damped, this will change slowly, and so can be assumed to be a constant value over one cycle, the value of which is obtained from the response measurement. The presence of $f_{D}(\theta)$ within the integral means that the application of this general formulation to a particular system depends on the functional form of nonlinearity present. Meskell [11] describes the formulation for a system with cubic damping, while the current work is concerned with a Coulomb nonlinearity.

3.2. Formulation for Coulomb Damping. Consider a singledegree-of-freedom system with both viscous (linear) and dry friction (Coulomb) damping. The damping force is given by

$$
f_{d}=c \dot{x}+\alpha \operatorname{sign}(\dot{x})= \begin{cases}c \dot{x}+\alpha, & \dot{x}>0, \\ 0, & \dot{x}=0, \\ c \dot{x}-\alpha, & \dot{x}<0 .\end{cases}
$$


Note that the damping coefficients are already mass normalized. Assuming a constant response amplitude of $A_{c}$, the work done over one period of vibration is

$$
\begin{aligned}
\int_{0}^{2 \pi} f_{D}(\theta) \dot{x}(\theta) d \theta= & c A_{c}^{2} \omega^{2} \int_{0}^{2 \pi} \cos ^{2} \theta d \theta \\
& +\alpha A_{c} \omega\left[\int_{-\pi / 2}^{\pi / 2} \cos \theta d \theta-\int_{\pi / 2}^{3 \pi / 2} \cos \theta d \theta\right] \\
= & \pi \omega^{2} c A_{c}^{2}+4 \omega \alpha A_{c} .
\end{aligned}
$$

Combining this with (10) yields

$$
A_{1}^{2}=A_{0}^{2}-\lambda_{1} A_{c}-\lambda_{2} A_{c}^{2},
$$

where

$$
\begin{aligned}
& \lambda_{1}=\frac{8}{\omega^{2}} \alpha, \\
& \lambda_{2}=\frac{2 \pi}{\omega} c .
\end{aligned}
$$

Using successive peaks of the free response, two sets of "identification coordinates" can now be defined.

For $A_{c}=A_{0}$,

$$
p_{2}=p_{1}\left(1-\lambda_{2}\right)-\lambda_{1}
$$

where the quantities $p_{1}$ and $p_{2}$ are defined as

$$
\begin{aligned}
& p_{1}=A_{0}, \\
& p_{2}=\frac{A_{1}^{2}}{A_{0}} .
\end{aligned}
$$

While for $A_{c}=A_{1}$,

$$
q_{2}=q_{1}\left(1+\lambda_{2}\right)+\lambda_{1}
$$

where the quantities $q_{1}$ and $q_{2}$ are defined as

$$
\begin{aligned}
& q_{1}=A_{1}, \\
& q_{2}=\frac{A_{0}^{2}}{A_{1}} .
\end{aligned}
$$

Fitting a straight line to these quantities will yield both an overestimate (from (15)) and an underestimate (from (17)) of the system parameters $\lambda_{1}$ and $\lambda_{2}$.

It is worth emphasizing that the form of the quantities $p_{i}$ and $q_{i}$ and (15) and (17) depend on the form of the nonlinearity in the system. To illustrate this, Table 3 compares the current formulation for Coulomb damping with that previously obtained for cubic damping [11].

\section{Results}

Figures 6 and 7 show the $p_{i}$ and $q_{i}$ quantities for modes 1 and 2 calculated from the filtered free response of $x_{1}$ shown in Figure 3. As can be seen, the data for both modes follows a

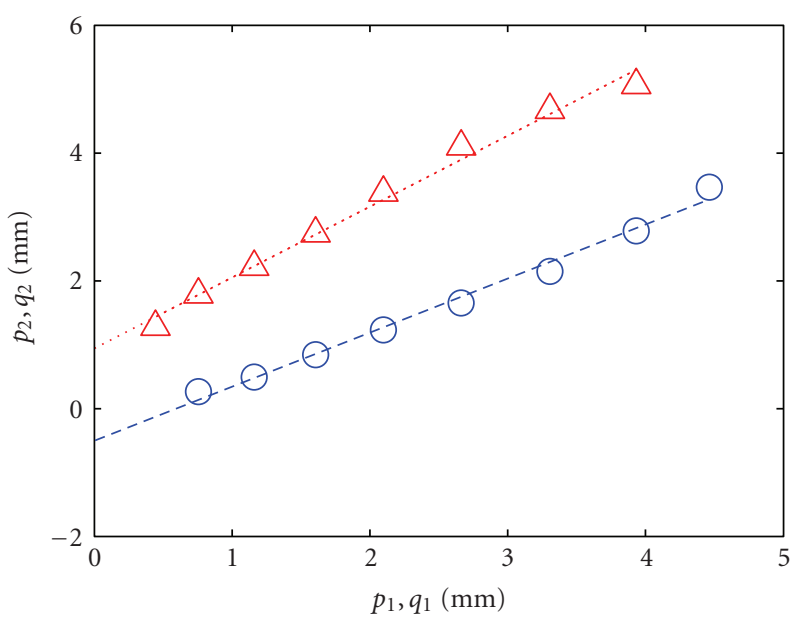

$\triangle q_{i}$

Figure 6: Mode 1 identification coordinates: $\bigcirc: p_{i} ; \triangle: q_{i}$.

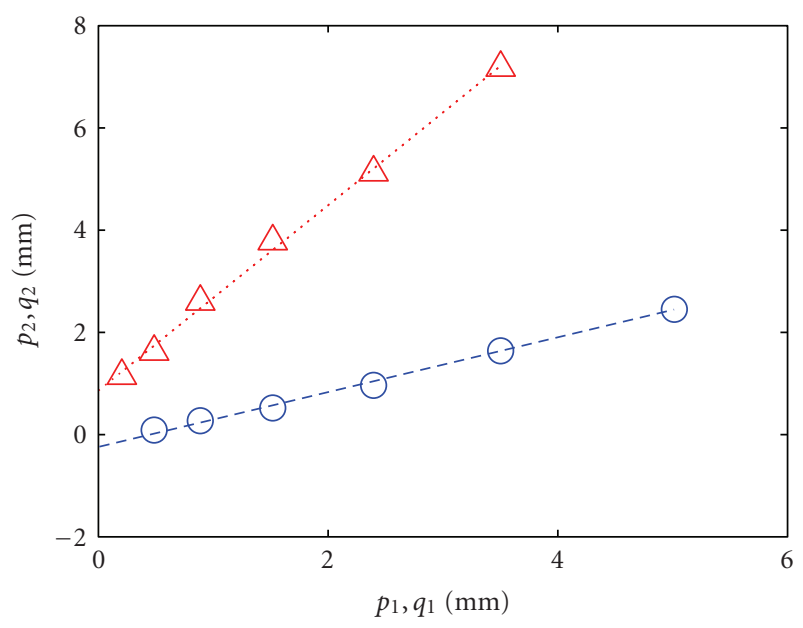

$\triangle q_{i}$

$\bigcirc p_{i}$

Figure 7: Mode 2 identification coordinates: $\bigcirc: p_{i} ; \Delta: q_{i}$.

straight line with non-zero intercept (i.e., $\lambda_{1} \neq 0$ ) indicating that both modes exhibit Coulomb damping.

An estimate of the mass-normalized damping parameters can now be obtained directly from the slope and intercept of the fitted line through each data set using (15) and (17), and the definitions of $\lambda_{i}$ given by Table 3 . This will yield an underand overestimate of the damping parameters for each mode. Table 4 details the average of the under- and overestimates for each mode.

\section{Model Validation}

In order to demonstrate the validity of this identification approach, the response of the fully coupled 2-degreeof-freedom system is compared with that obtained from 
TABLE 3: Comparison of formulations for two nonlinear systems.

\begin{tabular}{|c|c|c|c|c|c|c|}
\hline & $\lambda_{1}$ & $\lambda_{2}$ & $p_{2}$ & $p_{1}$ & $q_{2}$ & $q_{1}$ \\
\hline cubic $[11]$ & $\frac{2 \pi}{\omega} c$ & $\frac{3 \pi \omega}{2} \beta$ & $\left(\frac{A_{1}}{A_{0}}\right)^{2}$ & $-A_{0}^{2}$ & $\left(\frac{A_{0}}{A_{1}}\right)^{2}$ & $A_{1}^{2}$ \\
\hline Coulomb & $\frac{8}{\omega^{2}} \alpha$ & $\frac{2 \pi}{\omega} c$ & $\frac{A_{1}^{2}}{A_{0}}$ & $A_{0}$ & $\frac{A_{0}^{2}}{A_{1}}$ & $A_{1}$ \\
\hline
\end{tabular}

TABLE 4: Identified damping parameters.

\begin{tabular}{lcc}
\hline & $\alpha_{i}$ & $\zeta_{i}$ \\
\hline Mode 1 & 0.174 & 0.011 \\
Mode 2 & 0.890 & 0.050 \\
\hline
\end{tabular}

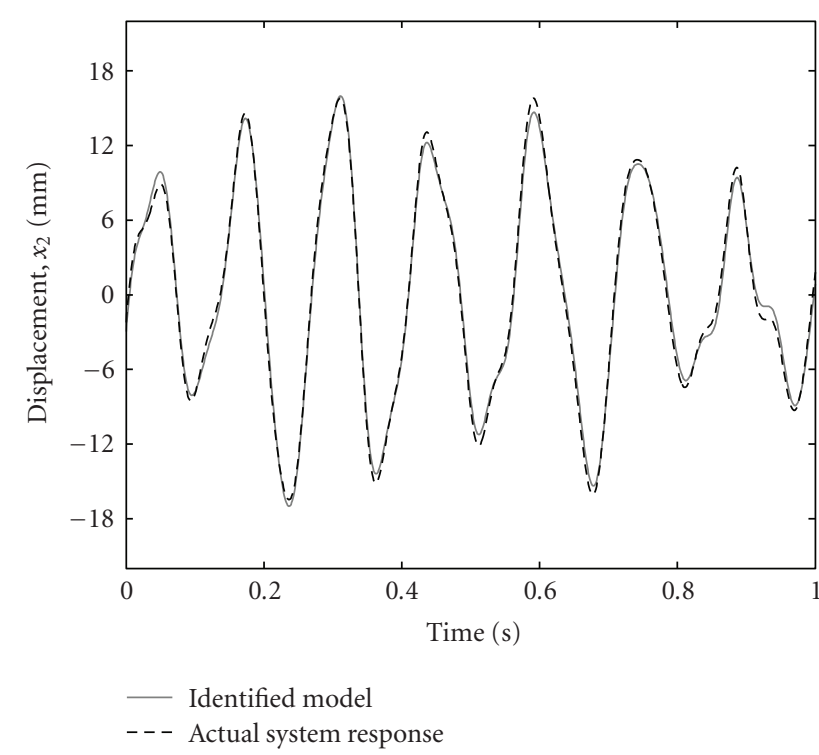

FIGURE 8: Forced response of mass 2 at an $\mathrm{rms}$ excitation level of $10 \mathrm{~N}$.

combining the response of the two identified single-degreeof-freedom systems. A random excitation force is applied to mass 1, as shown in Figure 1. This is band limited to $64 \mathrm{~Hz}$, so both natural frequencies will be excited, and various excitation levels have been examined. The excitation forces, $f_{i}$, for the single-degree-of-freedom systems, are obtained from the modal matrix

$$
[f]=[v][F],
$$

where $[v]$ is the modal matrix given by $(1)$ and $[F]=$ $\left[F_{1} 0\right]^{\prime}$. The displacement time histories of the 2 degrees of freedom can be obtained from the single-degree-of-freedom responses using the same modal matrix.

A section of the time response of mass 2 obtained from the 2-degree-of-freedom simulation is compared in Figure 8 with the corresponding time record obtained from the two single-degree-of-freedom systems. As can be seen, the behaviour of the identified model agrees well with that of the

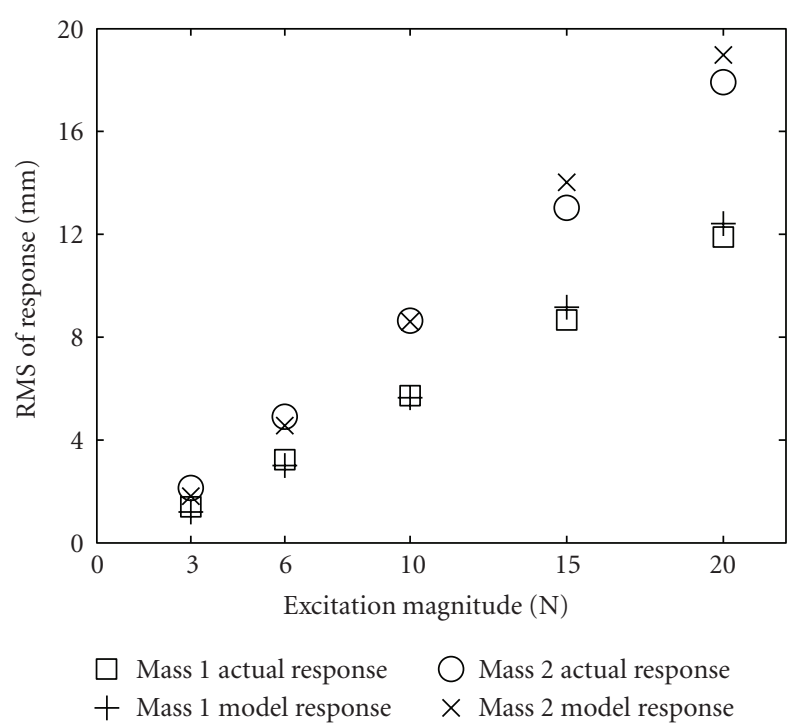

Figure 9: Forced response system at various excitation levels $\square$ : mass 1 actual response; +: mass 1 model response; $\bigcirc$ : mass 2 actual response; $\times$ : mass 2 model response.

original system. Similar results can be seen for the response of mass 1 and at other excitation levels.

Figure 9 shows the predicted rms values for each mass compared with the actual response for a range of excitation levels. As can be seen, the identified model tends to underestimate the system response at low excitation amplitudes, indicating that the effect of the nonlinear damping is systematically underestimated. Nonetheless, the identified singledegree-of-freedom models do provide reasonable agreement with the actual system response.

\section{Conclusions}

The feasibility of decomposing a lightly damped multidegree of freedom system with weak nonlinearity into a number of single-degree-of-freedom systems has been investigated in the context of a discrete 2 dof system. It has been shown that the free response at each mode can be obtained from a single free response measurement by band-pass filtering at each natural frequency. It has also been shown that the resulting free decay traces exhibit nonlinear damping and that it is possible to extract quantified damping parameters for each mode. Importantly, as the nonlinear behaviour is accounted for directly, the damping parameters obtained are 
independent of response amplitude. This would not be the case if a simple apparent linear damping was estimated. In the case of Coulomb damping, such an approach is likely to yield an overestimate of the operational damping ratio, and so an underestimate of vibration amplitudes. Further work is required to demonstrate the practicality of the method on experimental data and specifically to assess the range of parameter values for which the limiting assumptions are acceptable. Nonetheless, the method presented here is a promising technique for system identification in fluidcoupled systems.

\section{References}

[1] C. Meskell and J. A. Fitzpatrick, "Investigation of the nonlinear behaviour of damping controlled fluidelastic instability in a normal triangular tube array," Journal of Fluids and Structures, vol. 18, no. 5, pp. 573-593, 2003.

[2] H. Rice and J. Fitzpatrick, "The measurement of non-linear damping in single degree of freedom systems," Journal of Vibration and Acoustics, vol. 113, pp. 132-140, 1991.

[3] S. Granger, "A new signal processing method for investigating fluidelastic phenomena," Journal of Fluids and Structures, vol. 4, no. 1, pp. 73-97, 1990.

[4] S. Masri and T. Caughey, "A non-parametric identification technique for nonlinear dynamic problems," Journal of Applied Mechanics, vol. 46, pp. 433-447, 1979.

[5] S. F. Masri, R. K. Miller, A. F. Saud, and T. K. Caughey, "Identification of nonlinear vibrating structures-part I: formulation," Journal of Applied Mechanics, vol. 54, no. 4, pp. 918-922, 1987.

[6] S. F. Masri, R. K. Miller, A. F. Saud, and T. K. Caughey, "Identification of nonlinear vibrating structures-part II: applications," Journal of Applied Mechanics, vol. 54, no. 4, pp. 923929, 1987.

[7] K. Worden, "Data processing and experiment design for the restoring force surface method. Part 1: integration and differentiation of measured time data. Part 2: choice of excitation signal," Mechanical Systems and Signal Processing, vol. 4, pp. 295-344, 1990.

[8] C. Meskell, J. A. Fitzpatrick, and H. J. Rice, "Application of force-state mapping to a non-linear fluid-elastic system," Mechanical Systems and Signal Processing, vol. 15, no. 1, pp. 75-85, 2001.

[9] G. Kerschen, K. Worden, A. F. Vakakis, and J. C. Golinval, "Past, present and future of nonlinear system identification in structural dynamics," Mechanical Systems and Signal Processing, vol. 20, no. 3, pp. 505-592, 2006.

[10] C. Meskell and J. Fitzpatrick, "Errors in parameter estimates from the force state mapping technique for free response due to phase distortion," Journal of Sound and Vibration, vol. 252, no. 5, pp. 967-974, 2002.

[11] C. Meskell, "A decrement method for quantifying nonlinear and linear damping parameters," Journal of Sound and Vibration, vol. 296, no. 3, pp. 643-649, 2006.

[12] B. F. Feeny and J. W. Liang, "A decrement method for the simultaneous estimation of Coulomb and viscous friction," Journal of Sound and Vibration, vol. 195, no. 1, pp. 149-154, 1996.

[13] J. E. Mottershead and R. Stanway, "Identification of nth-power velocity damping," Journal of Sound and Vibration, vol. 105, no. 2, pp. 309-319, 1986.
[14] P. Eret and C. Meskell, "A practical approach to parameter identification for a lightly damped, weakly nonlinear system," Journal of Sound and Vibration, vol. 310, no. 4-5, pp. 829-844, 2008.

[15] M. Feldman, "Non-linear system vibration analysis using hilbert transform-I: free vibration analysis method 'Freevib', Mechanical Systems and Signal Processing, vol. 8, no. 2, pp. 119-127, 1994. 

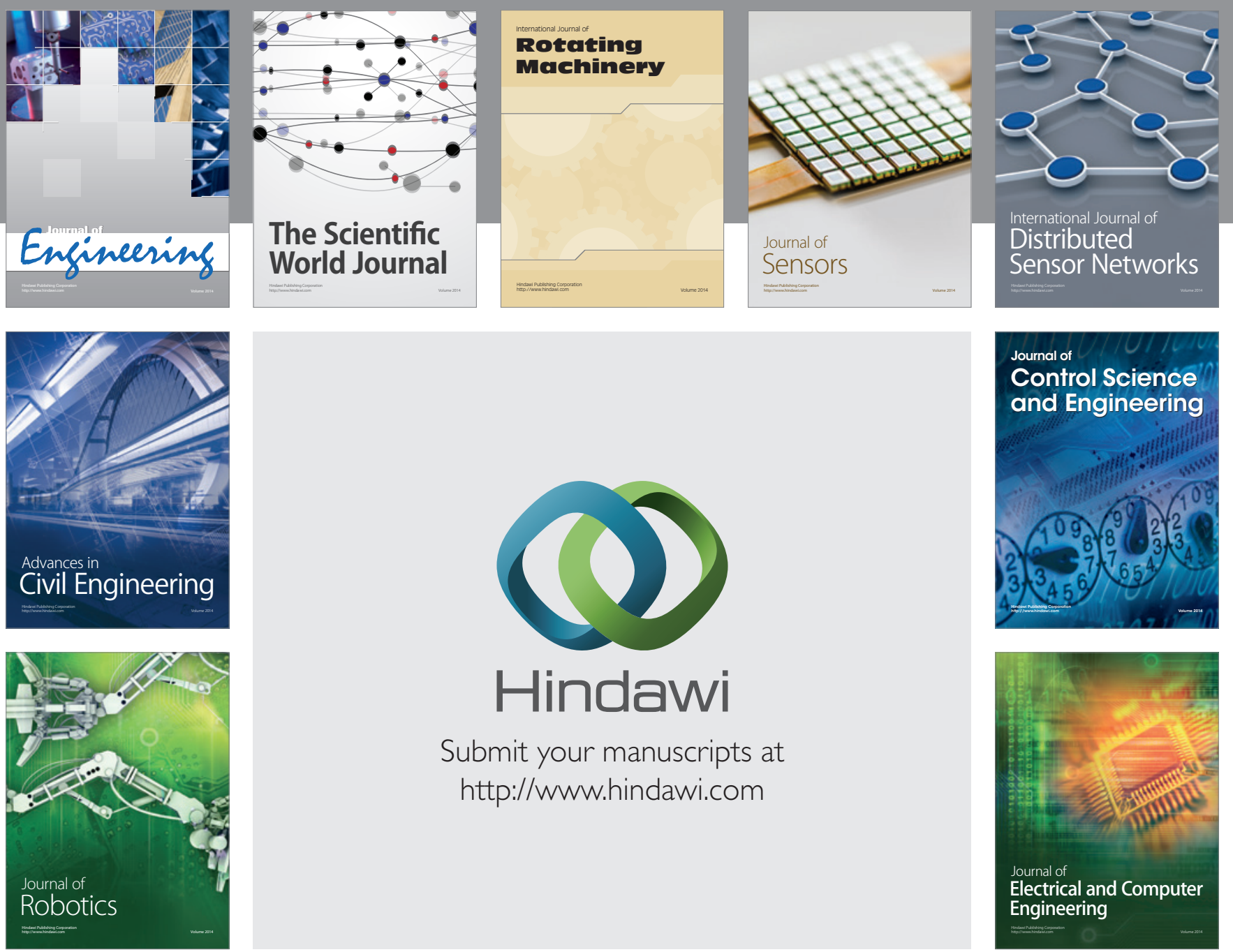

Submit your manuscripts at

http://www.hindawi.com
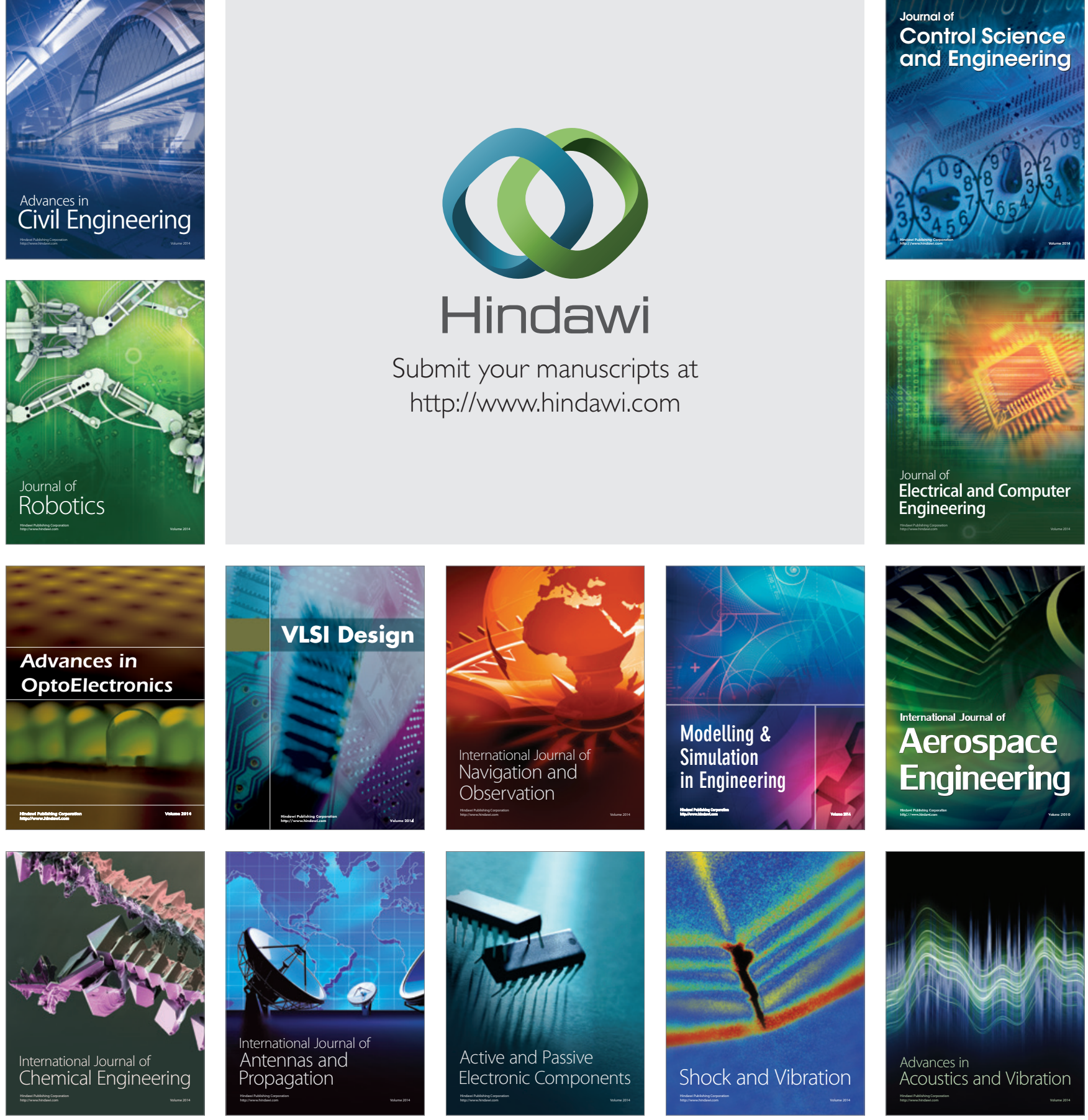